\title{
Semidirect Products and Invariant Connections
}

\author{
INDRANIL BISWAS
}

\begin{abstract}
Let $S$ be a complex reductive group acting holomorphically on a complex Lie group $N$ via holomorphic automorphisms. Let $K(S) \subset S$ be a maximal compact subgroup. The semidirect product $G:=N \rtimes K(S)$ acts on $N$ via biholomorphisms. We give an explicit description of the isomorphism classes of $G$-equivariant almost holomorphic hermitian principal bundles on $N$. Under the assumption that there is a central subgroup $Z=\mathrm{U}(1)$ of $K(S)$ that acts on $\operatorname{Lie}(N)$ as multiplication through a single nontrivial character, we give an explicit description of the isomorphism classes of $G$-equivariant holomorphic hermitian principal bundles on $N$.
\end{abstract}

Keywords: Semidirect product, holomorphic hermitian bundle, invariant connection, parabolic subgroup.

\section{Introduction}

Let $N$ and $S$ be connected complex Lie groups with $S$ acting holomorphically on $N$ via automorphisms. The semidirect product $N \rtimes S$ acts holomorphically on the complex manifold $N$. Our starting point is the observation that the holomorphic principal $S$-bundle

$$
N \rtimes S \longrightarrow(N \rtimes S) / S=N
$$

has a tautological flat holomorphic connection.

Received February 18, 2014.

2000 Mathematics Subject Classification. 53B35, 32L05

Supported by a J. C. Bose fellowship. 
Assuming that $S$ is reductive, fix a maximal compact subgroup $K(S)$ of it, and define

$$
G:=N \rtimes K(S) \subset N \rtimes S .
$$

Let $H$ be a complex connected reductive group and $K$ a maximal compact subgroup of it. A hermitian structure on a principal $H$-bundle $E_{H}$ over $N$ is a $C^{\infty}$ reduction of structure group of $E_{H}$ to $K$.

Our aim here is to study the $G$-equivariant (almost) holomorphic hermitian principal $H$-bundles on $N$.

Take a homomorphism $\beta: K(S) \longrightarrow K$. The action of $K(S)$ on $N$ produces an action of $K(S)$ on the Lie algebra $\mathfrak{n}$ of $N$. On the other hand, using $\beta$, the adjoint action of $K$ on its Lie algebra $\mathfrak{k}$ produces an action of $K(S)$ on $\mathfrak{k}$. Combining these, we get an action of $K(S)$ on

$$
\mathcal{W}:=\operatorname{Hom}_{\mathbb{R}}(\mathfrak{n}, \mathfrak{k})
$$

Consider all pairs $(\beta, \omega)$, where

- $\beta: K(S) \longrightarrow K$ is a homomorphism, and

- $\omega \in \mathcal{W}^{K(S)} \subset \mathcal{W}$ is an invariant.

Two such pairs $(\beta, \omega)$ and $\left(\beta^{\prime}, \omega^{\prime}\right)$ are called equivalent if there is an element $k \in K$ such that

- $\beta^{\prime}(g)=k \beta(g) k^{-1}$ for all $g \in K(S)$, and

- $\omega^{\prime}(v)=\operatorname{Ad}(k)((\omega)(v))$ for all $v \in \mathfrak{n}$.

Let $\mathcal{C}$ denote the set of equivalence classes of all such pairs.

We prove the following (see Lemma 4.3):

Lemma 1.1. The set of isomorphism classes of equivariant almost holomorphic hermitian principal $H$-bundle over $N$ is in bijection with $\mathcal{C}$.

The proof of Lemma 1.1 uses the tautological flat connection mentioned at the beginning. The bijection in Lemma 1.1 is very explicit. It is described in the proof of Theorem 5.1 .

Now assume that there is a central subgroup $Z=\mathrm{U}(1)$ of $K(S)$ that acts on the Lie algebra $\mathfrak{n}$ as multiplication through a single nontrivial character of $Z$. 
Since $\mathfrak{h}=\mathfrak{k} \otimes \mathbb{C}$ (recall that $H$ is reductive), we have $\mathcal{W}=\operatorname{Hom}_{\mathbb{C}}(\overline{\mathfrak{n}}, \mathfrak{h})$, where $\overline{\mathfrak{n}}$ is the conjugate of $\mathfrak{n}$. Any $\mathbb{C}$-linear map

$$
\alpha: \overline{\mathfrak{n}} \longrightarrow \mathfrak{h}
$$

produces a linear map $\bigwedge^{2} \alpha: \bigwedge^{2} \overline{\mathfrak{n}} \longrightarrow \bigwedge^{2} \mathfrak{h}$. Composing $\bigwedge^{2} \alpha$ with the Lie bracket $\bigwedge^{2} \mathfrak{h} \longrightarrow \mathfrak{h}$, we get

$$
\varphi(\alpha): \bigwedge^{2} \overline{\mathfrak{n}} \longrightarrow \mathfrak{h} .
$$

Define

$$
\mathcal{C}_{0}:=\{(\beta, \omega) \in \mathcal{C} \mid \varphi(\omega)=0\} \subset \mathcal{C} .
$$

We prove the following (see Theorem 5.1):

Theorem 1.2. The set of isomorphism classes of equivariant holomorphic hermitian principal $H$-bundle over $N$ is in bijection with $\mathcal{C}_{0}$.

\section{Semidirect products and a tautological connection}

\subsection{Action of a semidirect product}

Let $N$ be a connected complex Lie group. The group of all holomorphic automorphisms of the group $N$ that are connected to the identity map of $N$ will be denoted by $\operatorname{Aut}(N)$. In other words, $\operatorname{Aut}(N)$ is the connected component, containing the identity element, of the group of holomorphic automorphisms of $N$. So $\operatorname{Aut}(N)$ is a connected complex Lie group. Let $S$ be a connected complex affine algebraic group and

$$
\eta: S \longrightarrow \operatorname{Aut}(N)
$$

a holomorphic homomorphism of Lie groups. Let $N \rtimes S$ be the corresponding semidirect product. The underlying set for $N \rtimes S$ is $N \times S$, and the group structure on it is defined by the rule

$$
\left(u_{1}, g_{1}\right) \cdot\left(u_{2}, g_{2}\right)=\left(u_{1} \eta\left(g_{1}\right)\left(u_{2}\right), g_{1} g_{2}\right) .
$$

The complex Lie group $N \rtimes S$ acts on the complex manifold $N$ as follows:

$$
(u, g)(v)=u \eta(g)(v),
$$

where $(u, g) \in N \times S$ and $v \in N$. This action is clearly holomorphic; it does not preserve the group structure of $N$. 


\subsection{A connection}

Consider the complex subgroup $S \subset N \rtimes S$ given by the subset $\left\{e_{N}\right\} \times S \subset$ $N \times S$, where $e_{N}$ is the identity element of $N$. The projection

$$
N \rtimes S \longrightarrow(N \rtimes S) / S
$$

is a holomorphic principal $S$-bundle. Since

$$
(u, g)\left(e_{N}, g_{1}\right)=\left(u, g g_{1}\right),
$$

for all $u \in N$ and $g, g_{1} \in S$, the projection $N \rtimes S \longrightarrow N$ defined by $(u, g) \longmapsto u$ factors through the quotient $(N \rtimes S) / S$. The resulting map

$$
(N \rtimes S) / S \longrightarrow N
$$

is a biholomorphism. We will show that the holomorphic principal $S$-bundle in (2.3) has a natural flat holomorphic connection.

Let $\mathfrak{n}$ and $\mathfrak{p}$ be the Lie algebras of $N$ and $N \rtimes S$ respectively. Since $N$ is a normal subgroup of $N \rtimes S$, we conclude that $\mathfrak{n}$ is an ideal of $\mathfrak{p}$. The holomorphic tangent bundle of $N \rtimes S$ will be denoted by $T^{1,0}(N \rtimes S)$. Let

$$
\mathcal{H} \subset T^{1,0}(N \rtimes S)
$$

be the holomorphic subbundle obtained by translating the above mentioned subspace $\mathfrak{n} \subset \mathfrak{p}$ using the left-translation action of $N \rtimes S$ on itself. Since $\mathfrak{n}$ is an ideal in $\mathfrak{p}$, the right-translation action of $S$ on $N \rtimes S$ preserves this subbundle $\mathcal{H}$. It can be shown that $\mathcal{H}$ is a direct summand of the holomorphic subbundle of $T^{1,0}(N \rtimes S)$ given by the orbits of the righttranslation action of $S$ on $N \rtimes S$. Indeed, the two subbundles of $T^{1,0}(N \rtimes S)$ are clearly transversal at the identity element. Since both the subbundles are preserved by the left-translation action of $N \rtimes S$ on itself, they are transversal everywhere.

Since $\mathcal{H}$ is preserved by the right-translation action of $S$ on $N \rtimes S$, and it is a direct summand of the holomorphic subbundle of $T^{1,0}(N \rtimes S)$ given by the orbits of the right-translation action of $S$, there is a connection on the principal $S$-bundle in 2.3 whose horizontal distribution coincides with $\mathcal{H}$. Clearly, this condition uniquely determines the connection. The connection on the principal $S$-bundle in 2.3 constructed this way will be denoted by $\nabla^{S}$.

Since $\mathfrak{n}$ is closed under the Lie bracket operation on $\mathfrak{p}$, the distribution $\mathcal{H}$ in $(2.4)$ is integrable. Therefore, the above connection $\nabla^{S}$ is flat. The 
connection $\nabla^{S}$ is holomorphic because the distribution $\mathcal{H} \subset T^{1,0}(N \rtimes S)$ is holomorphic.

\section{Equivariant holomorphic hermitian bundles}

Henceforth, we assume that the group $S$ is reductive complex linear algebraic group.

Any two maximal compact subgroups of $S$ are conjugate by an element of $S$ (see [He, p. 256, Theorem 2.2(ii)]). Fix a maximal compact subgroup

$$
K(S) \subset S
$$

Define the subgroup

$$
G:=N \rtimes K(S) \subset N \rtimes S .
$$

In other words, $G$ is the subset $N \times K(S)$ of $N \times S$ which is in fact a Lie subgroup of $N \rtimes S$. The group $G$ acts on $N$ using the rule given in (2.2).

The group of biholomorphisms of the complex manifold $N$ will be denoted by $\operatorname{Hol}(N)$. Let

$$
\tau: G \longrightarrow \operatorname{Hol}(N)
$$

be the homomorphism defined by the above action.

\subsection{Equivariant hermitian principal bundles}

Let $H$ be a connected reductive linear algebraic group defined over $\mathbb{C}$. Fix a maximal compact subgroup

$$
K \subset H .
$$

Definition 3.1. A hermitian structure on a $C^{\infty}$ principal $H$-bundle $E_{H}$ over $N$ is a $C^{\infty}$ reduction of structure group

$$
E_{K} \subset E_{H}
$$

to the subgroup $K$ in 3.3 .

Let $\mathfrak{h}$ be the Lie algebra of $H$. Let $E_{H}$ be a $C^{\infty}$ principal $H$-bundle on $N$. It's adjoint vector bundle $E_{H} \times{ }^{H} \mathfrak{h}$ will be denoted by $\operatorname{ad}\left(E_{H}\right)$. 
Consider the Hodge type decomposition $\left(T^{*} N\right) \otimes_{\mathbb{R}} \mathbb{C}=\Omega_{N}^{1,0} \oplus \Omega_{N}^{0,1}$. The space of all connections on the principal $H$-bundle $E_{H}$ is an affine space for the vector space $C^{\infty}\left(N ; \operatorname{ad}\left(E_{H}\right) \otimes\left(\Omega_{N}^{1,0} \oplus \Omega_{N}^{0,1}\right)\right)$. Two connections $\nabla_{1}$ and $\nabla_{2}$ on the principal $H$-bundle $E_{H}$ are called equivalent if

$$
\nabla_{1}-\nabla_{2} \in C^{\infty}\left(N ; \operatorname{ad}\left(E_{H}\right) \otimes \Omega_{N}^{1,0}\right) \subset C^{\infty}\left(N ; \operatorname{ad}\left(E_{H}\right) \otimes\left(\Omega_{N}^{1,0} \oplus \Omega_{N}^{0,1}\right)\right) .
$$

An almost holomorphic structure on $E_{H}$ is an equivalence class of connections on $E_{H}[$ Ko, p. 87, Proposition 2].

The curvature of a connection $\nabla$ on $E_{H}$ will be denoted by $\mathcal{K}(\nabla)$. The component of $\mathcal{K}(\nabla)$ of Hodge type $(0,2)$ will be denoted by $\mathcal{K}(\nabla)^{0,2}$. If two connections $\nabla_{1}$ and $\nabla_{2}$ on $E_{H}$ are equivalent, then clearly we have $\mathcal{K}\left(\nabla_{1}\right)^{0,2}=\mathcal{K}\left(\nabla_{2}\right)^{0,2}$. The almost holomorphic structure on $E_{H}$ defined by a connection $\nabla$ on $E_{H}$ is integrable if and only if

$$
\mathcal{K}(\nabla)^{0,2}=0
$$

(see [Ko, p. 87, Proposition 3]). An integrable almost holomorphic structure on $E_{H}$ is a holomorphic structure on the principal $H$-bundle $E_{H}$.

An almost holomorphic hermitian principal $H$-bundle over $N$ is a triple $\left(E_{H}, \nabla, E_{K}\right)$, where $\left(E_{H}, \nabla\right)$ is an almost holomorphic principal $H$-bundle over $N$, and $E_{K} \subset E_{H}$ is a hermitian structure on $E_{H}$.

We will often suppress the notation for the equivalence class of connections; so when we say that $E_{H}$ is an almost holomorphic principal $H$-bundle we mean that $E_{H}$ is equipped with an equivalence class of connections.

An isomorphism from an almost holomorphic hermitian principal $H-$ bundle $\left(E_{H}, E_{K}\right)$ to an almost holomorphic hermitian principal $H$-bundle $\left(E_{H}^{\prime}, E_{K}^{\prime}\right)$ is a $C^{\infty}$ isomorphism of principal $H$-bundles

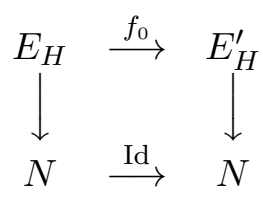

such that

- $f_{0}$ takes the almost holomorphic structure on $E_{H}$ to that of $E_{H}^{\prime}$, and

- $f_{0}\left(E_{K}\right)=E_{K}^{\prime}$.

An almost holomorphic hermitian principal $H$-bundle over $N$ whose almost complex structure is integrable is called a holomorphic hermitian 
principal $H$-bundle. An isomorphism between two holomorphic hermitian principal $H$-bundles is an isomorphisms of the underlying almost holomorphic hermitian principal $H$-bundles.

Now we consider the action of $G$ on $N$ (see (3.1) and (2.2)).

Definition 3.2. An equivariant hermitian principal $H$-bundle over $N$ is a triple of the form $\left(E_{H}, E_{K} ; \rho\right)$, where $f: E_{H} \longrightarrow N$ is a $C^{\infty}$ principal $H$-bundle, $E_{K} \subset E_{H}$ is a hermitian structure, and

$$
\rho: G \times E_{H} \longrightarrow E_{H}
$$

is a $C^{\infty}$ left-action of the group $G$ on $E_{H}$, satisfying the following conditions:

1) $f \circ \rho(g, z)=\tau(g)(f(z))$ for all $z \in E_{H}$ and $g \in G$, where $\tau$ is defined in 3.2 ,

2) the actions of $G$ and $H$ on $E_{H}$ commute, and

3) $\rho\left(G \times E_{K}\right)=E_{K}$.

The first two of the above three conditions mean that $z \longmapsto \rho(g, z)$ is a $C^{\infty}$ isomorphism of the principal $H$-bundle $E_{H}$ with the pulled back principal $H$-bundle $\tau\left(g^{-1}\right)^{*} E_{H}$. The last condition implies that this isomorphism between $E_{H}$ and $\tau\left(g^{-1}\right)^{*} E_{H}$ takes $E_{K}$ to $\tau\left(g^{-1}\right)^{*} E_{K}$.

An isomorphism between two equivariant hermitian principal $H$-bundles $\left(E_{H}, E_{K} ; \rho\right)$ and $\left(E_{H}^{\prime}, E_{K}^{\prime} ; \rho^{\prime}\right)$ over $N$ is a $C^{\infty}$ isomorphism of principal $H-$ bundles

$$
f_{0}: E_{H} \longrightarrow E_{H}^{\prime}
$$

such that $f_{0}\left(E_{K}\right)=E_{K}^{\prime}$, and $f_{0}(\rho(g, z))=\rho^{\prime}\left(g, f_{0}(z)\right)$ for all $g \in G$ and $z \in E_{H}$.

Let $\left(E_{H}, E_{K} ; \rho\right)$ be an equivariant hermitian principal $H$-bundle over $N$. A connection $\nabla$ on the principal $K$-bundle $E_{K}$ is called invariant if the action of $G$ on $E_{K}$ given by $\rho$ preserves $\nabla$. In other words, $\nabla$ is invariant if and only if for every $g \in G$, the isomorphism of $E_{K}$ with $\tau\left(g^{-1}\right)^{*} E_{K}$ defined by $z \longmapsto \rho(g, z)$ takes $\nabla$ to the pulled back connection $\tau\left(g^{-1}\right)^{*} \nabla$ on $\tau\left(g^{-1}\right)^{*} E_{K}$.

Definition 3.3. An equivariant almost holomorphic hermitian principal $H$-bundle over $N$ is an equivariant hermitian principal $H$-bundle $\left(E_{H}, E_{K} ; \rho\right)$ such that $E_{H}$ is equipped with an almost holomorphic structure satisfying the following condition: for each $g \in G$, the diffeomorphism 
of $E_{H}$ defined by $z \longmapsto \rho(g, z)$ preserves the almost complex structure on $E_{H}$.

An isomorphism between two equivariant almost holomorphic hermitian principal $H$-bundles $\left(E_{H}, E_{K} ; \rho\right)$ and $\left(E_{H}^{\prime}, E_{K}^{\prime} ; \rho^{\prime}\right)$ over $N$ is an isomorphism of equivariant hermitian principal $H$-bundles

$$
f_{0}: E_{H} \longrightarrow E_{H}^{\prime}
$$

that takes the almost complex structure on $E_{H}$ to that on $E_{H}^{\prime}$.

Lemma 3.4. Let $\left(E_{H}, E_{K} ; \rho\right)$ be an equivariant almost holomorphic hermitian principal $H$-bundle on $N$. Then there is a unique connection $\nabla^{K}$ on the principal $K$-bundle $E_{K}$ satisfying the following two conditions:

1) The connection $\nabla^{H}$ on $E_{H}$ induced by $\nabla^{K}$ lies in the equivalence class defining the almost holomorphic structure on $E_{H}$, and

2) the connection $\nabla^{K}$ is invariant.

Proof. Since $H$ is complex reductive, there is a unique connection $\nabla^{K}$ on the principal $K$-bundle $E_{K}$ such that the connection $\nabla^{H}$ on $E_{H}$ induced by $\nabla^{K}$ lies in the equivalence class defining the almost holomorphic structure on $E_{H}$ [At, pp. 191-192, Proposition 5]. From the uniqueness of the connection $\nabla^{K}$ it follows immediately that it is invariant.

\section{Equivariant almost holomorphic hermitian principal bundles}

Consider $G$ defined in (3.1). We note that $G / K(S)=(N \rtimes S) / S=N$. Therefore, the quotient map

$$
G \longrightarrow G / K(S)=N
$$

defines a reduction of structure group of the principal $S$-bundle in 2.3 to the subgroup $K(S) \subset S$. For any $g \in N \rtimes S$, the leaf of the foliation $\mathcal{H}$ in (2.4) passing through the point $g$ is $g N$. Therefore, if $g \in G$, then the leaf passing through $g$ is contained in $G$. This implies that the connection $\nabla^{S}$ constructed in Section 2.2 produces a connection on the principal $K(S)$ bundle $G \longrightarrow N$ in 4.1 ). This induced connection on the $K(S)$-bundle is flat because $\nabla^{S}$ is so. 
Since the principal $K(S)$-bundle in (4.1) is a reduction of structure group of the holomorphic principal $S$-bundle in 2.3 to the maximal compact subgroup $K(S) \subset S$, and $S$ is reductive, there is a unique connection $\nabla^{K(S)}$ on the principal $K(S)$-bundle in (4.1) such that the connection on the holomorphic principal $S$-bundle in $(2.3)$ induced by $\nabla^{K(S)}$ gives the almost holomorphic structure of it [At, pp. 191-192, Proposition 5].

Lemma 4.1. The above connection $\nabla^{K(S)}$ coincides with the flat connection on the principal $K(S)$-bundle $G \longrightarrow N$ induced by $\nabla^{S}$. In particular, $\nabla^{K(S)}$ is flat.

Proof. Since $\nabla^{S}$ induces a connection on the principal $K(S)$-bundle in (4.1), and $\nabla^{S}$ is holomorphic, in particular it is complex, the lemma follows from the uniqueness of $\nabla^{K(S)}$.

The Lie algebra of $K$ will be denoted by $\mathfrak{k}$. Let

$$
\mathcal{W}:=\operatorname{Hom}_{\mathbb{R}}(\mathfrak{n}, \mathfrak{k})
$$

be the space of all $\mathbb{R}$-linear homomorphisms from the vector space $\mathfrak{n}$ to the vector space $\mathfrak{k}$. We emphasize that the elements of $\mathcal{W}$ need not be Lie algebra homomorphisms.

The action of $K(S)$ on $N$ (see (2.1)) produces an action of $K(S)$ on $\mathfrak{n}$. Given a homomorphism $K(S) \longrightarrow K$ (see (3.3) for $K$ ), the adjoint action of $K$ on $\mathfrak{k}$ produces an action of $K(S)$ on $\mathfrak{k}$. Therefore, given a homomorphism $K(S) \longrightarrow K$, combining the actions of $K(S)$ on $\mathfrak{n}$ and $\mathfrak{k}$, we get an action of $K(S)$ on $\mathcal{W}$ defined in 4.2 .

Consider all pairs

$$
(\beta, \omega),
$$

where

- $\beta: K(S) \longrightarrow K$ is a homomorphism, and

- $\omega \in \mathcal{W}^{K(S)} \subset \mathcal{W}$.

The action of $K(S)$ on $\mathcal{W}$ is constructed as above, and $\mathcal{W}^{K(S)}$ is the space of invariants. Two such pairs $(\beta, \omega)$ and $\left(\beta^{\prime}, \omega^{\prime}\right)$ are called equivalent if there is an element $k \in K$ such that

- $\beta^{\prime}(g)=k \beta(g) k^{-1}$ for all $g \in K(S)$, and 
- $\omega^{\prime}(v)=\operatorname{Ad}(k)((\omega)(v))$ for all $v \in \mathfrak{n}$, where

$$
\operatorname{Ad}(k): \mathfrak{k} \longrightarrow \mathfrak{k}
$$

is the automorphism corresponding to the automorphism of $K$ defined by $x \longmapsto k x k^{-1}$.

Let

\section{$\mathcal{C}$}

denote the set of equivalence classes of pairs $(\beta, \omega)$ of the above type.

Consider all quadruples of the form

$$
\left(\left(E_{H}, E_{K} ; \rho\right), \nabla^{K}\right),
$$

where $\left(E_{H}, E_{K} ; \rho\right)$ is an equivariant hermitian principal $H$-bundle on $N$, and $\nabla^{K}$ is an invariant connection on $E_{K}$. Two such objects $\left(\left(E_{H}, E_{K} ; \rho\right), \nabla^{K}\right)$ and $\left(\left(E_{H}^{\prime}, E_{K}^{\prime} ; \rho^{\prime}\right), \nabla^{\prime}\right)$ are called isomorphic if there is an isomorphism of equivariant hermitian principal $H$-bundles between $\left(E_{H}, E_{K} ; \rho\right)$ and $\left(E_{H}^{\prime}, E_{K}^{\prime} ; \rho^{\prime}\right)$ that takes the connection $\nabla$ to $\nabla^{\prime}$.

Proposition 4.2. The set of isomorphism classes of all quadruples $\left(\left(E_{H}, E_{K} ; \rho\right), \nabla^{K}\right)$ of the above type is in bijection with the set $\mathcal{C}$ in (4.5).

Proof. Consider the flat connection $\nabla^{K(S)}$ on the principal $K(S)$-bundle $G \longrightarrow N$ (see Lemma 4.1). The left-translation action of $G$ on itself clearly preserves this connection. Indeed, this follows immediately from the fact that the distribution $\mathcal{H}$ in $(2.4)$ is preserved by the left-translation action of $N \rtimes S$ on itself. Note that by Lemma 4.1, the connection $\nabla^{K(S)}$ coincides with the one induced by the connection $\nabla^{S}$ that $\mathcal{H}$ defines.

Take any pair $(\beta, \omega)$ as in 4.3$)$. Let $E_{K}$ be the principal $K$-bundle on $N$ obtained by extending the structure group of the principal $K(S)$ bundle $G \longrightarrow N$ using the homomorphism $\beta$. The connection $\nabla^{K(S)}$ on the principal $K(S)$-bundle $G \longrightarrow N$ induces a connection on the associated bundle $E_{K}$. This induced connection on the principal $K$-bundle $E_{K}$ will be denoted by $\nabla^{\prime}$. Note that the total space of $E_{K}$ is the quotient of $G \times K$ where two points $\left(g_{1}, k_{1}\right)$ and $\left(g_{2}, k_{2}\right)$ are identified if there is an element $g \in$ $K(S)$ such that $g_{2}=g_{1} g$ and $k_{2}=\beta(g)^{-1} k_{1}$. Therefore, the left-translation action of $G$ on $G \times K$ descends to an action of $G$ on the quotient space $E_{K}$. This action of $G$ on $E_{K}$ clearly commutes with the action of $K$ on 
the principal $K$-bundle $E_{K}$. Also, the action of $G$ on $E_{K}$ preserves the connection $\nabla^{\prime}$ because the action of $G$ on the the principal $K(S)$-bundle $G \longrightarrow N$ preserves the connection $\nabla^{K(S)}$.

Let $\operatorname{ad}\left(E_{K}\right)=E_{K} \times{ }^{K} \mathfrak{k}$ be the adjoint vector bundle for $E_{K}$. The action of $G$ on $E_{K}$ defines an action on $\operatorname{ad}\left(E_{K}\right)$. This action of $G$ on $E_{K}$ and the action of $G$ on $N$ together produce an action of $G$ on the vector bundle $\operatorname{ad}\left(E_{K}\right) \otimes T^{*} N$, where $T^{*} N$ is the real cotangent vector bundle on $N$. Consider fiber $\left(\operatorname{ad}\left(E_{K}\right) \otimes T^{*} N\right)_{e_{N}}$ of $\operatorname{ad}\left(E_{K}\right) \otimes T^{*} N$ over the identity element $e_{N}$. We will show that it is canonically identified with $\operatorname{Hom}_{\mathbb{R}}(\mathfrak{n}, \mathfrak{k})$.

The fiber $T_{e_{N}}^{*} N$ is identified with $\mathfrak{n}^{*}$. The fiber $\left(E_{K}\right)_{e_{N}}$ is canonically identified with $K$ by sending any $k \in K$ to the equivalence class of $\left(e_{N}, k\right)$ (recall that $E_{K}$ is a quotient of $G \times K$ ). The identification between $\left(E_{K}\right)_{e_{N}}$ and $K$ produces an isomorphism between $\operatorname{ad}\left(E_{K}\right)_{e_{N}}$ and $\mathfrak{k}$ by sending any $v \in \mathfrak{k}$ to the equivalence class of $\left(e_{K}, v\right) \in K \times \mathfrak{k}$, where $e_{K}$ is the identity element of $K$; the vector bundle $\operatorname{ad}\left(E_{K}\right)$ is a quotient of $E_{K} \times \mathfrak{k}$, and using the identification of $K$ with $\left(E_{K}\right)_{e_{N}}$, the identity element $e_{K}$ gives an element of $\left(E_{K}\right)_{e_{N}}$. Therefore, the fiber $\left(\operatorname{ad}\left(E_{K}\right) \otimes T^{*} N\right)_{e_{N}}$ is canonically identified with $\mathfrak{n}^{*} \otimes \mathfrak{k}=\operatorname{Hom}_{\mathbb{R}}(\mathfrak{n}, \mathfrak{k})$.

Using the above identification of $\operatorname{Hom}_{\mathbb{R}}(\mathfrak{n}, \mathfrak{k})$ with $\left(\operatorname{ad}\left(E_{K}\right) \otimes T^{*} N\right)_{e_{N}}$, the element $\omega \in \operatorname{Hom}_{\mathbb{R}}(\mathfrak{n}, \mathfrak{k})$ gives an element of $\left(\operatorname{ad}\left(E_{K}\right) \otimes T^{*} N\right)_{e_{N}}$. Since $\omega$ is fixed by the action of $K(S)$, there is a unique $G$-invariant $C^{\infty}$ section $\widetilde{\omega}$ of $\operatorname{ad}\left(E_{K}\right) \otimes T^{*} N$ such that

$$
\widetilde{\omega}\left(e_{N}\right)=\omega .
$$

Consider the connection $\nabla^{\prime}+\widetilde{\omega}$ on $E_{K}$. Since both $\nabla^{\prime}$ and $\widetilde{\omega}$ are preserved by the action of $G$, it follows immediately the connection $\nabla^{\prime}+\widetilde{\omega}$ is also preserved by the action of $G$.

Let $E_{H}$ be the principal $H$-bundle on $N$ obtained by extending the structure group of the principal $K$-bundle $E_{K}$ using the inclusion of $K$ in $H$. Consider the connection on $E_{H}$ induced by $\nabla^{\prime}+\widetilde{\omega}$. The $(0,1)$-type component of it produces a holomorphic structure on $E_{H}$ because the connection comes from a connection on $E_{K}$. The action of $G$ on $E_{K}$ produces an action of $G$ on the associated by bundle $E_{G}$. This action of $E_{G}$ will be denoted by $\rho$.

Therefore, the quadruple $\left(\left(E_{H}, E_{K} ; \rho\right), \nabla^{\prime}+\widetilde{\omega}\right)$ satisfies all the required conditions.

To construct in inverse map, take any quadruple $\left(\left(E_{H}, E_{K} ; \rho\right), \nabla^{K}\right)$ as in the lemma. Fix a point

$$
z_{0} \in\left(E_{K}\right)_{e_{N}}
$$


in the fiber over the identity element $e_{N}$. Let

$$
\beta: K(S) \longrightarrow K
$$

be the map defined by $\rho\left(g, z_{0}\right)=z_{0} \beta(g), g \in K(S)$. We have

$$
\begin{gathered}
z_{0} \beta(g h)=\rho\left(g h, z_{0}\right)=\rho\left(g, \rho\left(h, z_{0}\right)\right)=\rho\left(g, z_{0} \beta(h)\right) \\
=\rho\left(g, z_{0}\right) \beta(h)=z_{0} \beta(g) \beta(h) .
\end{gathered}
$$

Therefore, $\beta$ is a homomorphism.

Let $d: T_{z_{0}} E_{K} \longrightarrow T_{e_{N}} N=\mathfrak{n}$ be the differential, at $z_{0}$, of the natural projection of $E_{K}$ to $N$. Let $\mathcal{H}_{z_{0}} \subset T_{z_{0}} E_{K}$ be the horizontal subspace for the connection $\nabla^{K}$ on $E_{K}$. The restriction of the homomorphism $d$ to $\mathcal{H}_{z_{0}}$ is an isomorphism. Let

$$
d^{\prime}: \mathfrak{n} \stackrel{\sim}{\longrightarrow} \mathcal{H}_{z_{0}} \subset T_{z_{0}} E_{K}
$$

be the inverse of $\left.d\right|_{\mathcal{H}_{z_{0}}}$. We note that the action of $N$ on $E_{K}$ given by $\rho$ produces a homomorphism

$$
\delta: \mathfrak{n} \longrightarrow T_{z_{0}} E_{K}
$$

Now note that the image of the homomorphism

$$
d^{\prime}-\delta: \mathfrak{n} \longrightarrow T_{z_{0}} E_{K}
$$

lies in the kernel of the homomorphism $d$. The kernel of $d$ is identified with the Lie algebra $\mathfrak{k}$ because $\left(E_{K}\right)_{e_{N}}$ is an orbit of the free action of $K$ on $E_{K}$. Therefore, we have

$$
d^{\prime}-\delta: \mathfrak{n} \longrightarrow \mathfrak{k}
$$

In other words,

$$
\omega:=d^{\prime}-\delta \in \operatorname{Hom}_{\mathbb{R}}(\mathfrak{n}, \mathfrak{k}) .
$$

Since the connection $\nabla^{K}$ is preserved by the action of $G$, it follows that $\omega$ is fixed by the action of $K(S)$.

Therefore, $(\beta, \omega) \in \mathcal{C}$.

It is straightforward to check that the above two constructions are inverses of each other.

Lemma 4.3. The set of isomorphism classes of equivariant almost holomorphic hermitian principal $H$-bundle over $N$ is in bijection with $\mathcal{C}$ in (4.5). 
Proof. Using Lemma 3.4, the set of isomorphism classes of equivariant almost holomorphic hermitian principal $H$-bundle over $N$ is identified with set of the isomorphism classes of quadruples $\left(\left(E_{H}, E_{K} ; \rho\right), \nabla^{K}\right)$ in Proposition 4.2. Therefore, the lemma follows from Proposition 4.2 .

As before, the Lie algebra of $H$ will be denoted by $\mathfrak{h}$. Since $K$ is a maximal compact subgroup of the complex reductive group $H$, the inclusion of $\mathfrak{k}$ in $\mathfrak{h}$ produces a $\mathbb{C}$-linear isomorphism of $\mathfrak{k} \otimes_{\mathbb{R}} \mathbb{C}$ with $\mathfrak{h}$. Let $\overline{\mathfrak{n}}$ be the complex vector space conjugate to $\mathfrak{n}$. So the underlying real vector space for $\overline{\mathfrak{n}}$ is the underlying real vector space for $\mathfrak{n}$, but the multiplication by $\lambda \in \mathbb{C}$ on $\overline{\mathfrak{n}}$ is the multiplication by $\bar{\lambda}$ on $\mathfrak{n}$. Therefore, $\mathcal{W}$ (see (4.2)) has the following natural identification

$$
\mathcal{W}=\operatorname{Hom}_{\mathbb{C}}(\overline{\mathfrak{n}}, \mathfrak{h})=\mathfrak{h} \otimes_{\mathbb{C}} \overline{\mathfrak{n}}^{*} .
$$

This identification commutes with the actions of $G$.

\section{Equivariant holomorphic hermitian principal bundles}

Now assume that the connected component of the center of $K(S)$ containing the identity element is nontrivial. Fix a subgroup

$$
Z=\mathrm{U}(1) \subset K(S)
$$

contained in the center of $K(S)$.

Henceforth, we assume that there is a nontrivial character

$$
\chi_{0}: Z \longrightarrow \mathbb{C}^{*}
$$

such that the action of any $g \in Z$ on the Lie algebra $\mathfrak{n}$, given by $\eta$ in (2.1), is multiplication by $\chi_{0}(g)$.

Consider $\mathcal{W}=\operatorname{Hom}_{\mathbb{C}}(\overline{\mathfrak{n}}, \mathfrak{h})$ defined in 4.2 (see (4.6)). Any $\mathbb{C}$-linear map

$$
\alpha: \overline{\mathfrak{n}} \longrightarrow \mathfrak{h}
$$

produces a linear map $\bigwedge^{2} \alpha: \bigwedge^{2} \overline{\mathfrak{n}} \longrightarrow \bigwedge^{2} \mathfrak{h}$. Composing $\bigwedge^{2} \alpha$ with the Lie bracket $\Lambda^{2} \mathfrak{h} \longrightarrow \mathfrak{h}$, we get a $\mathbb{C}$-linear map

$$
\varphi(\alpha): \bigwedge^{2} \overline{\mathfrak{n}} \longrightarrow \mathfrak{h} .
$$

Define the subset of $\mathcal{C}$ (see 4.5p)

$$
\mathcal{C}_{0}:=\{(\beta, \omega) \in \mathcal{C} \mid \varphi(\omega)=0\} \subset \mathcal{C},
$$


where the map $\varphi$ is constructed in $(5.3)$.

Theorem 5.1. The set of isomorphism classes of equivariant holomorphic hermitian principal $H$-bundle over $N$ is in bijection with $\mathcal{C}_{0}$ defined in (5.4).

Proof. Take a homomorphism

$$
\beta: K(S) \longrightarrow K \text {. }
$$

Consider the principal $K(S)$-bundle $G \longrightarrow N$ in 4.1 . Let

$$
E_{K}:=G \times{ }^{K(S)} K \longrightarrow N
$$

be the principal $K$-bundle obtained by extending the structure group of it using the homomorphism $\beta$ in (5.5). Consider the connection $\nabla^{K(S)}$ on the principal $K(S)$-bundle in (4.1) (see Lemma 4.1). It induces a connection on the above associated principal $K$-bundle $E_{K}$. This induced connection on $E_{K}$ will be denoted by $\nabla^{K}$. This connection $\nabla^{K}$ is flat because $\nabla^{K(S)}$ is so.

The left-translation action of $G$ on itself produces a left-action of $G$ on $E_{K}$. To see this action, first note that $E_{K}$ is the quotient of $G \times K$ where two points $\left(g_{1}, k_{1}\right)$ and $\left(g_{2}, k_{2}\right)$ are identified if there is an element $z \in$ $K(S)$ such that $g_{2}=g_{1} z$ and $k_{2}=\beta(z)^{-1} k_{1}$. Therefore, the left-translation action of $G$ on $G \times K$ descends to a left-action of $G$ on $E_{K}$. The above connection $\nabla^{K}$ on $E_{K}$ is preserved by this action of $G$ on $E_{K}$. Indeed, this follows immediately from the fact that the connection $\nabla^{K(S)}$ is preserved by the left-translation action of $G$ on itself (see the proof of Proposition 4.2).

Let $E_{H}:=E_{K} \times{ }^{K} H \longrightarrow N$ be the principal $H$-bundle obtained by extending the structure group of the principal $K$-bundle $E_{K}$ using the inclusion of $K$ in $H$. Note that $E_{H}$ is identified with the principal $H$-bundle

$$
G \times{ }^{K(S)} H \longrightarrow N
$$

obtained by extending the structure group of the principal $K(S)$-bundle $G \longrightarrow N$ using the composition homomorphism

$$
K(S) \stackrel{\beta}{\longrightarrow} K \hookrightarrow H .
$$

The left-action of $G$ on $E_{K}$ produces a left-action of $G$ on $E_{H}$. This action of $G$ on $E_{H}$ will be denoted by $\rho$. The connection $\nabla^{K}$ on $E_{K}$ induces a connection on the associated bundle $E_{H}$. This induced connection on the 
principal $H$-bundle $E_{H}$ will be denoted by

$$
\nabla^{H}
$$

This connection $\nabla^{H}$ is flat because $\nabla^{K}$ is so. The flat connection $\nabla^{H}$ defines a holomorphic structure on the principal $H$-bundle $E_{H}$. We note that $\nabla^{H}$ is preserved by the above defined action $\rho$ of $G$ on $E_{H}$ because $\nabla^{K(S)}$ is preserved by the left-translation action of $G$ on itself. This implies that for each $g \in G$, the diffeomorphism of $E_{H}$ given by the action of $g$ is holomorphic.

Consequently,

$$
\left(E_{H}, E_{K} ; \rho\right)
$$

is an equivariant holomorphic hermitian principal $H$-bundle on $N$. It corresponds to the pair $(\beta, 0) \in \mathcal{C}$ by the bijection in Lemma 4.3

Now take an invariant element

$$
\omega \in \mathcal{W}^{K(S)}
$$

(see 4.2 and (4.6) for the action of $K(S)$ on $\mathcal{W}$ constructed using $\beta$ in (5.5). Let

$$
\operatorname{ad}\left(E_{H}\right)=E_{H} \times{ }^{H} \mathfrak{h} \longrightarrow N
$$

be the adjoint vector bundle for the principal $H$-bundle $E_{H}$ in (5.7). The fiber $\operatorname{ad}\left(E_{H}\right)_{e_{N}}$ is canonically identified with the Lie algebra $\mathfrak{h}$ (as before, $e_{N}$ is the identity element of $N$ ). To see this identification, first note that $\operatorname{ad}\left(E_{H}\right)$ is the quotient of $G \times H \times \mathfrak{h}$ where two elements $\left(g_{1}, h_{1}, v_{1}\right)$ and $\left(g_{2}, h_{2}, v_{2}\right)$ of $G \times H \times \mathfrak{h}$ are identified if there are elements $x \in K(S)$ and $h \in H$ such that $g_{2}=g_{1} x^{-1}, h_{2}=\beta(x) h_{1} h^{-1}$ and $v_{2}=\operatorname{Ad}(h)\left(v_{1}\right)$ (see (4.4) for $\operatorname{Ad}(h))$. The Lie algebra $\mathfrak{h}$ is identified with the fiber $\operatorname{ad}\left(E_{H}\right)_{e_{N}}$ by sending any $v \in \mathfrak{h}$ to the equivalence class of $\left(e, e_{H}, v\right)$, where $e$ and $e_{H}$ are the identity elements of $G$ and $H$ respectively.

Since the holomorphic tangent space $T_{e_{N}}^{1,0} N$ to $N$ at $e_{N}$ is identified with $\mathfrak{n}$, the anti-holomorphic tangent space $T_{e_{N}}^{0,1} N$ is identified with $\overline{\mathfrak{n}}$. in view of the above isomorphism of $\mathfrak{h}$ with $\operatorname{ad}\left(E_{H}\right)_{e_{N}}$, the vector space $\mathcal{W}$ in 4.6 gets identified with the space of $\mathbb{C}$-linear maps $\operatorname{Hom}_{\mathbb{C}}\left(T_{e_{N}}^{0,1} N, \operatorname{ad}\left(E_{H}\right)_{e_{N}}\right)$.

The action $\rho$ of $G$ on $E_{H}$ in (5.7) produces an action of $G$ on the adjoint vector bundle $\operatorname{ad}\left(E_{H}\right)$. Therefore, we get an action of the isotropy subgroup $K(S)$ on the fiber $\operatorname{ad}\left(E_{H}\right)_{e_{N}}$. The above identification between $\mathcal{W}$ and $\operatorname{Hom}_{\mathbb{C}}\left(T_{e_{N}}^{0,1} N, \operatorname{ad}\left(E_{H}\right)_{e_{N}}\right)$ clearly intertwines the actions of $K(S)$. 
Let

$$
\omega^{\prime} \in \operatorname{Hom}_{\mathbb{C}}\left(T_{e_{N}}^{0,1} N, \operatorname{ad}\left(E_{H}\right)_{e_{N}}\right)=\operatorname{ad}\left(E_{H}\right)_{e_{N}} \otimes \Omega_{N, e_{N}}^{0,1}=\operatorname{ad}\left(E_{H}\right)_{e_{N}} \otimes \overline{\mathfrak{n}}^{*}
$$

be the element that corresponds to $\omega$ in (5.8) by the above identification between $\mathcal{W}$ and $\operatorname{Hom}_{\mathbb{C}}\left(T_{e_{N}}^{0,1} N, \operatorname{ad}\left(E_{H}\right)_{e_{N}}\right)$. Since $\omega$ is fixed by the action of $K(S)$, this element $\omega^{\prime}$ is also fixed by the action of $K(S)$. Therefore, there is a unique $G$-invariant section

$$
\widetilde{\omega} \in C^{\infty}\left(N ; \operatorname{ad}\left(E_{K}\right) \otimes \Omega_{N}^{0,1}\right)^{G}
$$

such that $\widetilde{\omega}\left(e_{N}\right)=\omega^{\prime}$.

Consider the connection $\nabla^{H}$ on $E_{H}$ constructed in (5.6). Note that

$$
\widetilde{\nabla}^{H}:=\nabla^{H}+\widetilde{\omega}
$$

is a connection on $E^{H}$. Let $\widetilde{E}_{H}$ be the almost homomorphic principal $H$ bundle defined by this connection $\widetilde{\nabla}^{H}$. Therefore,

$$
\left(\widetilde{E}_{H}, E_{K} ; \rho\right)
$$

is an equivariant almost holomorphic hermitian principal $H$-bundle on $N$, where $E_{K}$ are $\rho$ are as in (5.7). This equivariant almost holomorphic hermitian principal $H$-bundle corresponds to the pair $(\beta, \omega)$ by the bijection in Lemma 4.3 .

Let $\mathcal{K}\left(\widetilde{\nabla}^{H}\right)$ denote the curvature of the above connection $\widetilde{\nabla}^{H}$ on $E_{H}$. The component of $\mathcal{K}\left(\widetilde{\nabla}^{H}\right)$ of Hodge type $(0,2)$ will be denoted by $\mathcal{K}\left(\widetilde{\nabla}^{H}\right)^{0,2}$. We note that the above almost holomorphic principal $H$-bundle $\widetilde{E}_{H}$ is holomorphic if and only if

$$
\mathcal{K}\left(\widetilde{\nabla}^{H}\right)^{0,2}=0
$$

(see $(3.4))$.

Let $\nabla$ be the connection on the adjoint vector bundle ad $\left(E_{H}\right)$ induced by the connection $\nabla^{H}$ on $E_{H}$ in (5.6). Since the connection $\nabla^{H}$ is flat, we have

$$
\mathcal{K}\left(\widetilde{\nabla}^{H}\right)^{0,2}=\widehat{\nabla}(\widetilde{\omega})^{0,2}+(\widetilde{\omega} \bigwedge \widetilde{\omega})^{0,2}
$$

where the superscript $(0,2)$ denotes the component of Hodge type $(0,2)$. 
We will show that

$$
\widehat{\nabla}(\widetilde{\omega})^{0,2}=0 .
$$

Let $Z^{*}:=\operatorname{Hom}(Z, \mathbb{C} \backslash\{0\})$ be the group of characters of the subgroup $Z$ in (5.1). Note that $Z^{*}$ is isomorphic to $\mathbb{Z}$. Consider the action of the isotropy subgroup $K(S)$ on the fiber $\operatorname{ad}\left(E_{H}\right)_{e_{N}}=\mathfrak{h}$ given by $\rho$ (the action of $K(S)$ on $\mathfrak{h}$ is given by the homomorphism $\beta$ ). Restrict this action of $K(S)$ to the subgroup $Z \subset K(S)$. Let

$$
\operatorname{ad}\left(E_{H}\right)_{e_{N}}=\bigoplus_{\chi \in Z^{*}} V^{\chi}
$$

be the isotypical decomposition of the $Z$-module. Since $Z$ is contained in the center of $K(S)$, the action of $K(S)$ on $\operatorname{ad}\left(E_{H}\right)_{e_{N}}$ preserves the decomposition in (5.13).

Take any character $\chi \in Z^{*}$. Since the subspace $V^{\chi} \subset \operatorname{ad}\left(E_{H}\right)_{e_{N}}$ is preserved by the action of the isotropy subgroup $K(S) \subset G$, there is a unique $C^{\infty}$ subbundle

$$
\mathcal{V}^{\chi} \subset \operatorname{ad}\left(E_{H}\right)
$$

such that

- $\mathcal{V}^{\chi}$ is preserved by the action of $G$ on $\operatorname{ad}\left(E_{H}\right)$, and

- the fiber $\mathcal{V}_{e_{N}}^{\chi}=V^{\chi}$

Since $\mathcal{V}^{\chi}$ is preserved by the action of $G$, it follows that $\mathcal{V}^{\chi}$ is preserved by the connection $\widehat{\nabla}$ (as before, $\widehat{\nabla}$ is the connection of ad $\left(E_{H}\right)$ induced by the connection $\nabla^{H}$ on $E_{H}$ ). Indeed, the condition that $\mathcal{V}^{\chi}$ is preserved by the action of $G$ implies that $\mathcal{V}^{\chi}$ is identified with the flat vector bundle $G \times{ }^{K(S)} V^{\chi}$ associated to the flat principal $K(S)$-bundle $G \longrightarrow N$ for the $K(S)$-module $\mathcal{V}_{e_{N}}^{\chi}=V^{\chi}$.

We recall that $\widetilde{\omega}\left(e_{N}\right)=\omega^{\prime} \in \operatorname{ad}\left(E_{H}\right)_{e_{N}} \otimes \Omega_{N, e_{N}}^{0,1}$ is fixed by the action of the isotropy subgroup $K(S)$. In particular, $\widetilde{\omega}\left(e_{N}\right)$ is fixed by the action of $Z \subset K(S)$. The group $Z$ acts on the complex vector space $\Omega_{N, e_{N}}^{0,1}=\overline{\mathfrak{n}}^{*}$ as multiplication through the character $\chi_{0}$ in (5.2). Consequently, we have

$$
\omega^{\prime} \in V^{\chi_{0}^{-1}} \otimes \Omega_{N, e_{N}}^{0,1}
$$

(see (5.13)). This implies that

$$
\widetilde{\omega} \in C^{\infty}\left(N ; \mathcal{V}^{\chi_{0}^{-1}} \otimes \Omega_{N}^{0,1}\right),
$$


because $\widetilde{\omega}$ is fixed by the action of $G$ and the subbundle $\mathcal{V}^{\chi_{0}^{-1}} \subset \operatorname{ad}\left(E_{H}\right)$ is preserved by the action of $G$. Therefore, we have

$$
\widehat{\nabla}(\widetilde{\omega})^{0,2} \in C^{\infty}\left(N ; \mathcal{V}^{\chi_{0}^{-1}} \otimes \Omega_{N}^{0,2}\right)^{G}
$$

(recall that the connection $\widehat{\nabla}$ is invariant under the action $\rho$ of $G$ on $E_{H}$ ). Therefore, the evaluation

$$
\widehat{\nabla}(\widetilde{\omega})^{0,2}\left(e_{N}\right) \in \mathcal{V}^{\chi_{0}^{-1}} \otimes \Omega_{N, e_{N}}^{0,2}
$$

is fixed under the action of $K(S)$, in particular, it is fixed by the action of the subgroup $Z$. But $Z$ acts on $\Omega_{N, e_{N}}^{0,2}$ as multiplication through the character $\chi_{0}^{2}$, because it acts on $\Omega_{N, e_{N}}^{0,1}$ as multiplication through the character $\chi_{0}$. Therefore, $Z$ acts on $V^{\chi_{0}^{-1}} \otimes \Omega_{N, e_{N}}^{0,2}$ as multiplication through the character $\chi_{0}$. Since $\chi_{0}$ is nontrivial, this implies that we have the space of invariants

$$
\left(V^{\chi_{0}^{-1}} \otimes \Omega_{N, e_{N}}^{0,2}\right)^{Z}=0 .
$$

In particular, we have $\widehat{\nabla}(\widetilde{\omega})^{0,2}\left(e_{N}\right)=0$. Therefore, $\widehat{\nabla}(\widetilde{\omega})^{0,2}=0$, because it is $G$-invariant. This proves $(5.12)$.

In view of (5.12), from (5.11) we conclude that

$$
\mathcal{K}\left(\widetilde{\nabla}^{H}\right)^{0,2}=(\widetilde{\omega} \bigwedge \widetilde{\omega})^{0,2} .
$$

Now, it is easy to see that $(\widetilde{\omega} \wedge \widetilde{\omega})^{0,2}\left(e_{N}\right)=\varphi(\omega)$, where $\varphi$ is constructed in (5.3). Therefore, we conclude that (5.10) holds if and only if $\varphi(\omega)=0$. This completes the proof.

\section{Examples}

Let $G_{0}$ be a simple linear algebraic group defined over $\mathbb{C}$. Let

$$
P \subsetneq G_{0}
$$

be a proper parabolic subgroup. The unipotent radical of $P$ will be denoted by $R_{u}(P)$. The quotient

$$
L^{\prime}(P):=P / R_{u}(P)
$$


is a connected reductive complex linear algebraic group. Fix a connected complex reductive subgroup

$$
L(P) \subset P
$$

such that the composition

$$
L(P) \hookrightarrow P \longrightarrow P / R_{u}(P)=L^{\prime}(P)
$$

is an isomorphism. Such a subgroup $L(P)$ is called a Levi factor of $P[\mathrm{Hu}$, p. 184]. We note that Levi factors of $P$ exist, and any two Levi factors of $P$ are conjugate by an element of $R_{u}(P)[\mathrm{Hu}$, p. 185, Theorem].

The Levi subgroup $L(P)$ has the adjoint action on $R_{u}(P)$. The group $P$ is identified with the corresponding semidirect product $R_{u}(P) \rtimes L(P)$ by sending any $(u, g) \in R_{u}(P) \times L(P)$ to $u g \in P$.

In the previous notation, $N=R_{u}(P)$ and $S=L(P)$.

Let $R_{n}(\mathfrak{p})$ denote the Lie algebra of the unipotent radical $R_{u}(P)$. Assume that $R_{n}(\mathfrak{p})$ is abelian. Then $P$ is a maximal proper parabolic subgroup. Therefore, the center of $L(P)$ is isomorphic to $\mathbb{C}^{*}$. Hence, the center of a maximal compact subgroup $K$ of $L(P)$ is isomorphic to $\mathrm{U}(1)$. The adjoint action of the center of $K$ on $R_{n}(\mathfrak{p})$ is multiplication through a single nontrivial character of the center.

All maximal proper parabolic subgroups of $\operatorname{SL}(n, \mathbb{C})$ satisfy the condition that the unipotent radical is abelian. $\operatorname{Both} \operatorname{Sp}(2 n, \mathbb{C})$ and $\operatorname{SO}(n, \mathbb{C})$ and also the exceptional groups have parabolic subgroups satisfying the the condition that the unipotent radical is abelian.

\section{References}

[At] M. F. Atiyah, Complex analytic connections in fibre bundles, Trans. Amer. Math. Soc. 85 (1957), 181-207.

[BT] I. Biswas and A. Teleman, Invariant connections and invariant holomorphic bundles on homogeneous manifolds, Cent. Euro. Jour. Math. 12 (2014), 1-13.

[He] S. Helgason, Differential Geometry, Lie Groups, and Symmetric Spaces, Graduate Studies in Mathematics, 34. American Mathematical Society, Providence, RI, 2001. 
[Hu] Humphreys, J. E., Linear algebraic groups, Graduate Texts in Mathematics, Vol. 21, Springer-Verlag, New York, Heidelberg, Berlin, 1987.

[Ko] J.-L. Koszul, Lectures on fibre bundles and differential geometry, Tata Institute of Fundamental Research Lectures on Mathematics, No. 20, T.I.F.R., Bombay, 1965; http://www.math.tifr.res.in/ publ/ln/tifr20.pdf.

Indranil Biswas

School of Mathematics,

Tata Institute of Fundamental Research,

Homi Bhabha Road, Bombay 400005,

India

E-mail: indranil@math.tifr.res.in 